\title{
Optimizing bandwidth of fiber optical parametric amplifier with different combinations of higher-order dispersion coefficient signs
}

\author{
N. Othman ${ }^{1}, N . S . M$. Shah $^{1, *}, K . G$. Tay $^{1}$, and N. A. Cholan ${ }^{1}$ \\ ${ }^{1}$ Faculty of Electrical and Electronic Engineering, Universiti Tun Hussein Onn Malaysia, 86400 Batu Pahat, Malaysia
}

\begin{abstract}
This paper investigates the influence of different combinations of second-order $\left(\beta_{2}\right)$, fourth-order $\left(\beta_{4}\right)$ and sixth-order $\left(\beta_{6}\right)$ dispersion coefficient signs (negativity/positivity) to the fiber optical parametric amplifier gain performance. The numerical simulation has exploited the fourth-order Runge-Kutta method to solve the coupled amplitude equations which basically represent the parametric process of four-wave mixing. In the normal regime at which $\beta_{2}$ is positive, the fiber optical parametric amplifier exhibits a poor gain spectrum and it is well performed once the pump is presumed to be in anomalous regime i.e. $\beta_{2}$ is negative. The effect of $\beta_{4}$ is significant when the signal wavelength is further from the pump wavelength and a wide amplification bandwidth is produced when the fiber have positive $\beta_{4}$ in the anomalous regime. As for $\beta_{6}$, unfortunately in this simulation its influences are hardly can be seen probably because of its small value regardless its sign, but, from phase-mismatch equation it is shown that its impact is prominent when the signal is positioned way far from the pump. All in all, this shown that the signs combination of the higher-order dispersion coefficients are significant in order to optimize the FOPA amplification bandwidth.
\end{abstract}

\section{Introduction}

The nonlinear effect of FWM has been utilized in many applications such as the parametric oscillators [1], FWMassisted lasers [2], FWM-cascaded [3], optical generators [4] and filter [5]. The ability of FWM effect to amplify a signal wave together with the generation of an idler wave made the effect convenient for those mentioned applications. One of the applications which also exploited the nonlinear effect of FWM is the fiber optical parametric amplifier (FOPA). The FOPA researches in the communication systems have greatly developed since its capability in providing adjustable center frequency and adjustable gain spectra have surpassed the conventional amplifiers i.e. Raman amplifier and Erbium-doped fiber amplifier [6].

There are several ways to optimize the FOPA gain spectrum for instance by increasing the pump power to obtain high parametric gain [7] and place the pump close to zero dispersion frequency to attain wide amplification bandwidth [8]. Either way, one can also include the higher-order dispersion coefficient of a fiber in order to optimize the FOPA gain spectrum. The higher-order dispersion coefficients have been proven to be important in obtaining the desire amplification bandwidth [9]. The involvement of dispersion coefficients up to sixth-order shows significant improvement of the amplification bandwidth [10]. However, the signs of these coefficients are needed to be well combined or else it will result in substandard gain spectrum. Hence, in this paper the effects of the combination of signs of the second-order, fourth-order and sixth-order dispersion coefficients to the FOPA gain spectrum were investigated. During the numerical simulation, the FOPA is assumed to be experience losses and the pump is depleted.

\section{Mathematical model}

The FOPA amplifies the signal by utilizing the effect of FWM which caused by the third-order nonlinear susceptibility. The coupled amplitude equations are given by [11]

$$
\begin{array}{r}
\frac{d A_{p}}{d z}=i \gamma\left[A_{p}\left(\left|A_{p}\right|^{2}+2\left(\left|A_{s}\right|^{2}+\left|A_{i}\right|^{2}\right)\right)\right] \\
+2 i \gamma A_{s} A_{i} A_{p}^{*} \exp (i \Delta \beta z), \\
\frac{d A_{s}}{d z}=i \gamma\left[A_{s}\left(\left|A_{s}\right|^{2}+2\left(\left|A_{p}\right|^{2}+\left|A_{i}\right|^{2}\right)\right)\right] \\
+i \gamma A_{i}^{*} A_{p}^{2} \exp (-i \Delta \beta z), \\
\frac{d A_{i}}{d z}=i \gamma\left[A_{i}\left(\left|A_{i}\right|^{2}+2\left(\left|A_{p}\right|^{2}+\left|A_{s}\right|^{2}\right)\right)\right] \\
+i \gamma A_{s}^{*} A_{p}^{2} \exp (-i \Delta \beta z),
\end{array}
$$

are basically represented the process of FWM, where $\gamma$ is the fiber nonlinearity and $A_{j}$ for $j \in\{p, s, i\}$ are the amplitudes of the pump, signal and idler, meanwhile $A_{j}^{*}$ are the corresponding complex conjugate. Noteworthy, the fiber attenuation can be taken into account by adding term $-(\alpha / 2) A_{j}$ to the right hand side of each equations.

\footnotetext{
* Corresponding author: shahida@uthm.edu.my
} 
The phase-mismatch, $\Delta \beta$ along the fiber length $z$ is generally expressed as [11]

$$
\Delta \beta=2 \sum_{m=1}^{\infty} \frac{\beta_{2 m}}{(2 m) !}(\Delta \omega)^{2 m},
$$

where $\Delta \omega=\omega_{p}-\omega_{s}$ is the frequency detuning and $\omega_{k}=$ $2 \pi c / \lambda_{k}$ for $k \in\{p, s\}$ are the frequency of pump and signal in terms of wavelength, $\lambda_{k}$. The odd order of dispersion coefficients do not contribute to the gain spectrum, that is why only even order are taken into account [11]. The quantity of $\beta_{2 m}=d^{2 m} \beta(\omega) / d \omega^{2 m}$ with $\beta$ as mode-propagation constant expanded by Taylor series, can be related to the higher-order dispersion coefficient calculated at zero dispersion frequency, $\omega_{0}$ such as [9]

$$
\beta_{2 m}=\sum_{n=2 m}^{\infty} \frac{\beta_{n 0}}{(n-2 m) !}\left(\omega_{p}-\omega_{0}\right)^{n-2 m} .
$$

Since this paper will investigate the linear phasemismatch curve with the involvement of second-order, fourth-order and sixth-order dispersion coefficient denoted by $\beta_{2}, \beta_{4}$ and $\beta_{6}$, respectively, thus by combining (4) and (5) for $m=1$ to 3 , the $\Delta \beta$ can be written as

$$
\begin{gathered}
\Delta \beta=\beta_{2}(\Delta \omega)^{2}+\frac{\beta_{4}}{3}\left[2\left(\omega_{p}-\omega_{0}\right)^{2}+\frac{1}{4}(\Delta \omega)^{2}\right](\Delta \omega)^{2} \\
+\frac{\beta_{6}}{24}\left[\left(\omega_{p}-\omega_{0}\right)^{4}+\frac{1}{15}(\Delta \omega)^{4}\right](\Delta \omega)^{2} .
\end{gathered}
$$

The $\beta_{2}$ sign is depends on the position of pump i.e. negative if the pump wavelength is in anomalous regime and positive if the pump wavelength is in normal regime. In practice, the positivity or negativity of the $\beta_{4}$ and $\beta_{6}$ at the respective pump wavelength are depend on the fiber dispersion profile. However, in this paper their signs are assumed to be negative or positive depending on the combination.

In order to compute the FOPA parametric gain, firstly, the Eqs. (1)-(3) were solved by using built-in function of Matlab 2014, ODE45 which basically adopting the numerical method named fourth-order Runge-Kutta. Then, the parametric gain for the respective wavelength was obtained by dividing the output with the input signal power such as

$$
\mathrm{G}(\mathrm{dB})=10 \log \left(\frac{P_{s, \text { out }}}{P_{s, \text { in }}}\right),
$$

and generally $P_{S}$ is the square of $A_{S}$ i.e $P_{S}=\left|A_{S}\right|^{2}$ for both input and output.

\section{Results and Discussion}

This section simulates the impacts of the different combinations of $\beta_{2}, \beta_{4}$ and $\beta_{6}$ signs on FOPA gain spectrum. The pump power, $\mathrm{P}_{\mathrm{p}}$ of $1 \mathrm{~W}$ was launched into the $500 \mathrm{~m}$ long highly-nonlinear dispersion-shifted fiber (HNL-DSF) to amplify the signal with the power of -40 $\mathrm{dBm}$. Note that, the HNL-DSF has the zero dispersion wavelength (ZDW) at $1556.5 \mathrm{~nm}$. The fiber attenuation and nonlinearity are $0.82 \mathrm{~dB} / \mathrm{km}$ and $11.5 \mathrm{~W}^{-1} \mathrm{~km}^{-1}$ correspondingly. Given that $\beta_{2}=1.970 \times 10^{-2} \mathrm{ps}^{2} / \mathrm{km}$, $\beta_{4}=6.231 \times 10^{-5} \mathrm{ps}^{4} / \mathrm{km}$ and $\beta_{6}=1.178 \times 10^{-8}$ $\mathrm{ps}^{6} / \mathrm{km}$, the sign of the respective higher-order coefficients were manipulated depending on the combinations. There are eight combinations of $\beta_{2}, \beta_{4}$ and $\beta_{6}$ signs [9]. These combinations are basically can be divided into two categories, firstly, when the pump is placed in the normal regime of HNL-DSF dispersion profile which results in positive value of $\beta_{2}$ and secondly, when the pump is lauched in the anomalous regime at which the $\beta_{2}$ value is negative. In order to effectively investigate the effects of the higher-order dispersion coefficients sign to the FOPA gain spectrum, the position of pump is fixed at $\lambda_{\mathrm{p}}=1558 \mathrm{~nm}$ and it is assumed to be in the normal or anomalous regimes, depending on the case of study.

Fig. 1 pictures the FOPA gain spectra when the pump is assumed to be in the normal regime. It is worth noting that since the total gain spectrum of FOPA is symmetric with respect to $\lambda_{\mathrm{p}}$, here the half gain spectra were plotted. It can be seen that FOPA experiences poor gain due to the domination of the positive $\beta_{2}$ value in (6) thus results in large phase-mismatch especially when the signal wavelength offsets further from the pump. Note that, the real parametric gain occurs when $\Delta \beta$ is lies in between $-4 \gamma P_{p}$ to 0 . In Fig. 2, the corresponding $\Delta \beta$ of the gain spectra in Fig. 1 were plotted against the $\lambda_{s}-\lambda_{p}$. Evidently, the reason of poor gains are because of the $\Delta \beta$ curve lies outside the proper area, especially the curves in Fig. 2(a) and Fig. 2(b). In addition, it is worth to mention that the positive $\beta_{4}$ is also contributing to this large phasemismatch. The positive values of both $\beta_{2}$ and $\beta_{4}$ have monopolized the $\Delta \beta$ and even the negative value of $\beta_{6}$ is unable to encounter it, which explain why the almost similar spectrum is obtained in Fig. 1(a) and Fig. 1(b). On the other hand, Fig. 2(c) and Fig. 2(d) show that with the negative value of $\beta_{4}$, some parts of $\Delta \beta$ curve lie within $-4 \gamma P_{p}$ and 0 notably when the $\lambda_{s}$ is far from the $\lambda_{p}$ which produces the parametric gain at the respective wavelength; see Fig. 1(c) and Fig. 1(d). Unfortunately, in these figures, the influence of $\beta_{6}$ is not clear which evidenced by the no changes of the resulted gain spectrum regardless the sign of $\beta_{6}$. This is probably because the

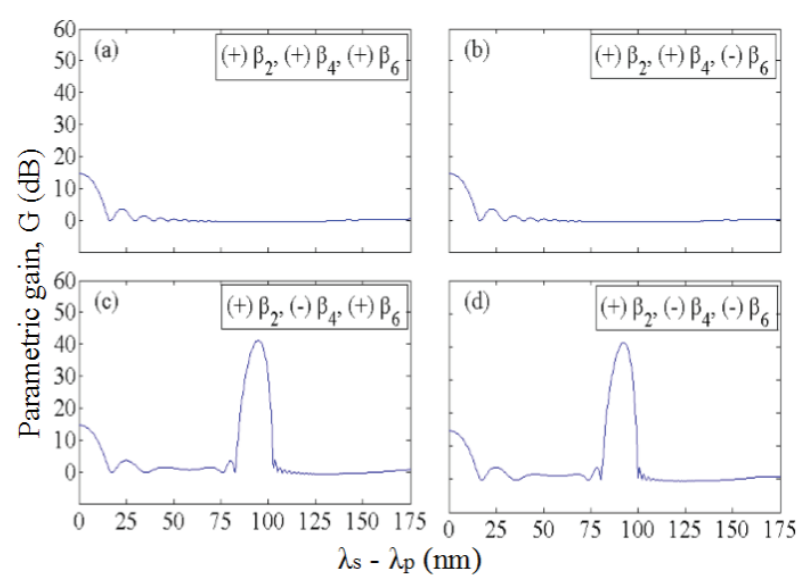

Fig. 1. Half gain spectrum of the FOPA when $\lambda_{p}$ is assumed to be in normal regime. 


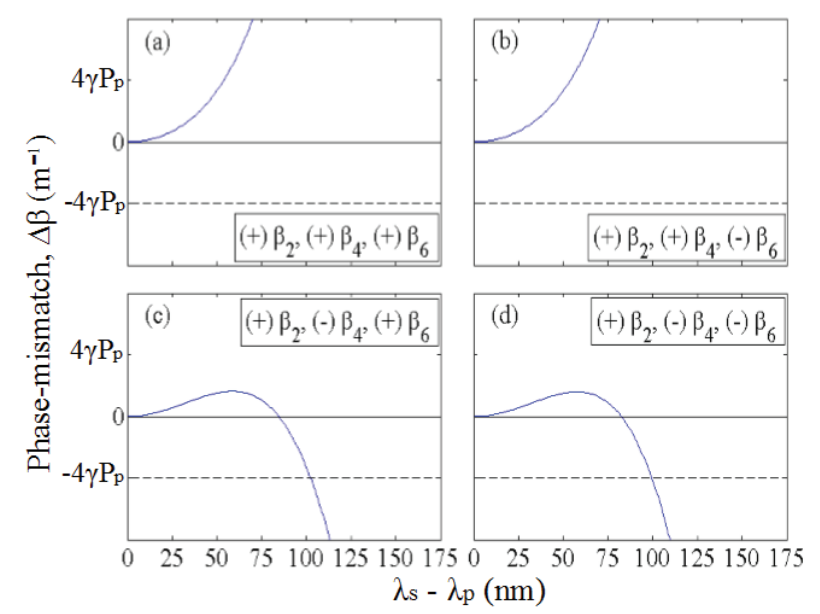

Fig. 2. The phase-mismatch of the FOPA gain spectrum corresponding to Fig. 1.

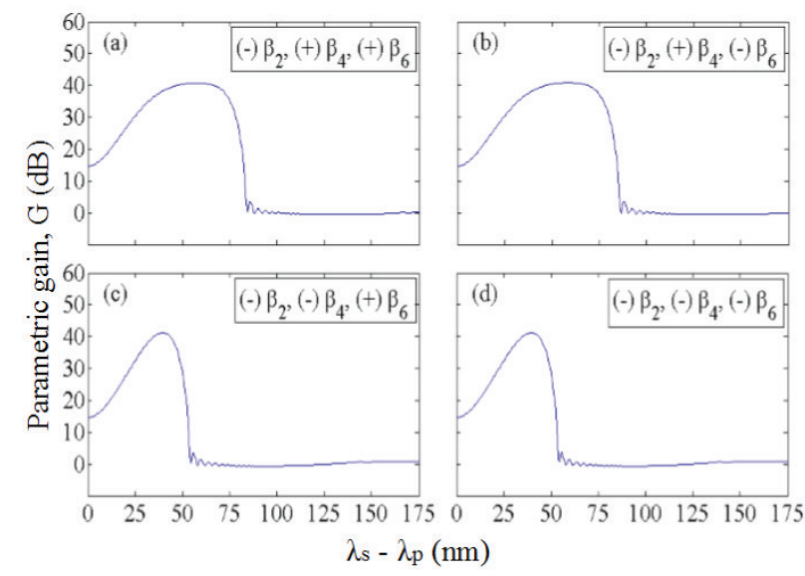

Fig. 3. Half gain spectrum of the FOPA when $\lambda_{p}$ is assumed to be in anomalous regime.

value of $\beta_{6}$ is very small and consequently gives no effect to $(6)$.

Next, in Fig. 3 the pump is presumed to be positioned in the anomalous regime in which the value of $\beta_{2}$ is negative. It exhibits that the FOPA can perform efficiently in this regime. The obtained amplification bandwidth implies that the $\Delta \beta$ definitely lies in the range of $-4 \gamma P_{p}$ to 0 and Fig. 4 has proven it. As seen in Fig. 3(a) and Fig. $3(\mathrm{~b})$, the positive value of $\beta_{4}$ causes the FOPA to amplify the signal at the larger $\lambda_{s}$ then results in the high parametric gain and subsequently the FOPA bandwidth. This also portrays that the value of $\beta_{4}$ is significant when the distance of pump and signal wavelength is large enough. Similar with previous resulted gain spectra, the changes of $\beta_{6}$ sign from positive to negative does not affect the $\Delta \beta$ curve. Additionally, in Fig. 4(c) and Fig. 4(d), the effect of $\beta_{6}$ regardless its sign has been suppressed by the domination of the negative values of both $\beta_{2}$ and $\beta_{4}$, which then results in almost similar spectrum in Fig. 3(c) and Fig. 3(d). Although the effects of $\beta_{6}$ to the FOPA gain spectra cannot be clearly seen in this simulation results, it is believed that if the value of $\beta_{6}$ is carefully tailored, the large amplification bandwidth is possibly obtained [10].

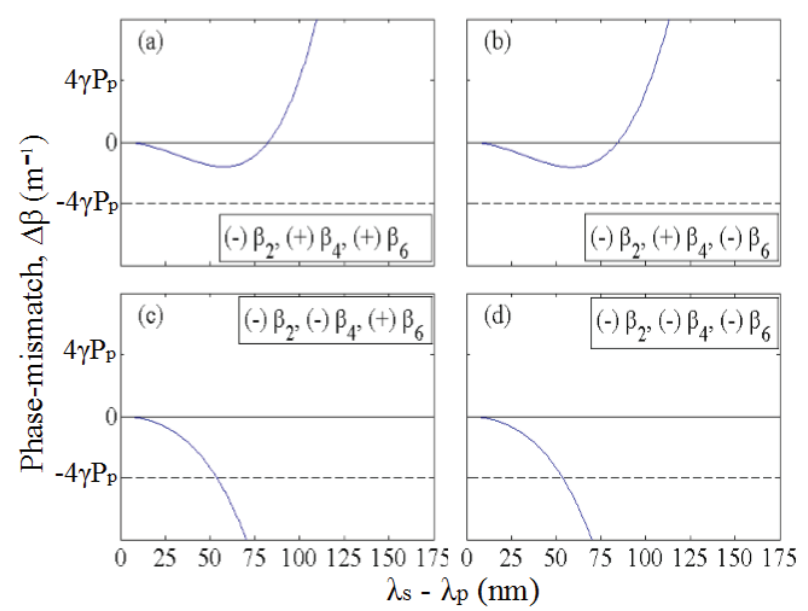

Fig. 4. The phase-mismatch of the FOPA gain spectrum corresponding to Fig. 3.

\section{Conclusion}

This paper has numerically investigated the effects of the different combination of $\beta_{2}, \beta_{4}$ and $\beta_{6}$ signs to the FOPA gain spectrum. In order to realize that, the well-known coupled amplitude equations have been solved by using fourth-order Runge-Kutta method in Matlab 2014. When the pump is placed in normal regime, the positive value of $\beta_{2}$ causes the large phase-mismatch and the consequence is worst if it is combined with the positive $\beta_{4}$. Nonetheless, the FOPA performance is practically works well in the anomalous regime in which $\beta_{2}$ is negative. This is because the phase-mismatch in this regime is small particularly when FOPA is operated close to ZDW. Noteworthy, the influence of $\beta_{4}$ and $\beta_{6}$ is more significant when signal is launched at the wavelength far from the pump. Yet, the effect of $\beta_{6}$ sign is hardly can be observed because of $\beta_{6}$ small value and the effect is almost disappeared if it is combined with both positive or both negative values of $\beta_{2}$ and $\beta_{4}$, since their impact are dominant in (6). To summarize, the combination of $\beta_{2}>$ $0, \beta_{4}>0$ and $\beta_{6}>0$ is resulted in a poor gain spectrum as the phase-mismatch is very large for this combination. Generally, the FOPA amplification bandwidth can be optimized if the values of $\beta_{2}, \beta_{4}$ and $\beta_{6}$ are carefully tailored and their signs combination are well chosen.

This work was supported in part by Centre for Graduate Studies Universiti Tun Hussein Onn Malaysia (UTHM) and Research Supporting Grant Scheme (RSGS) Vot U102 granted by UTHM.

\section{References}

1. B. Sun, K. Hu, D. Chen, Y. Wei, S. Gao, S. He, J. Light. Technol. 30, 1937 (2012)

2. N. A. Cholan, M. H. Al-Mansoori, A. S. M. Noor, A. Ismail, M. A. Mahdi, Appl. Phys. B Lasers Opt. 115, 251 (2014)

3. N. A. Cholan, M. H. Al-Mansoori, A. S. M. Noor, A. Ismail, M. A. Mahdi, Opt. Express 21, 6131 (2013) 
4. N. S. M. Shah, M. Matsumoto, Opt. Commun. 284, 4687 (2011)

5. D. Xu, Y. Cao, Z. R. Tong, J. P. Yang, Optoelectron. Lett. 12, 417 (2016)

6. M. E. Marhic, P. A. Andrekson, P. Petropoulos, S. Radic, C. Peucheret, M. Jazayerifar, Laser Photon. Rev. 9, 50 (2015)

7. H. Pakarzadeh, M. Taghizadeh, M. Hatami, J. Nonlinear Opt. Phys. Mater. 25, 1650023 (2016)

8. P. S. Maji, P. R. Chaudhuri, IEEE Photonics J. 7, 1 (2015)
9. S. K. Chatterjee, S. N. Khan, P. R. Chaudhuri, Opt. Commun. 332, 244 (2014)

10. P. Dainese, G. S. Wiederhecker, A. A. Rieznik, H. L. Fragnito, H. E. Hernández-Figueroa, SBMO/IEEE MTT-S International Microwave and Optoelectronics Conference Proceedings 2005, 92 (2005)

11. M. E. Marhic, Fiber Optical Parametric Amplifiers, Oscillators and Related Devices (Cambridge University Press, UK, 2008) 